\title{
Taking the bite out of TMJD pain
}

Temporomandibular joint disorder (TMJD) is characterized by severe jaw pain associated with chewing or biting down. There is no effective treatment for the disorder, which affects more than 10 million Americans. TMJD can arise from trauma, but for many cases, the underlying cause is not known, hindering attempts to develop therapeutic strategies. A study by researchers at Duke University (Durham, NC) has now identified a protein that is required for TMJDassociated pain in mice. The protein, TRPV4, may be a new target for pain relief in TMJD.

TRPV4 is an ion channel protein that allows calcium to rapidly enter cells. It is known to be involved in both inflammation and mechanically evoked pain. Wolfgang Liedtke, who led the study, explained why his team focused on TRPV4. "TRPV4 is widely expressed in sensory neurons found in the trigeminal ganglion, which is responsible for all sensations of the head, face and their associated structures, such as teeth, the tongue and temporomandibular joint," he said in a press release. "This

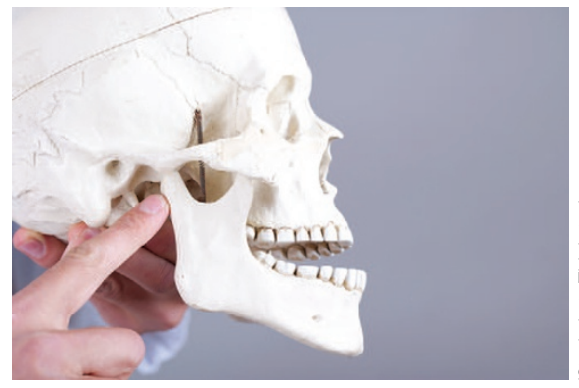

pattern and the fact that TRPV4 has been found to be involved in response to mechanical stimulation made it a logical target to explore."

Liedtke's team compared normal mice with mice in which the gene encoding TRPV 4 was ablated so that the protein could not be expressed. They injected complete Freund's adjuvant bilaterally into the TMJs of the mice to create inflammation as a model of TMJD and measured the bite force exerted by the mice. Because bite force lessens as TMJ pain increases, bite-force attenuation is a suitable proxy for assessing TMJD-like pain in the mice.
Mice lacking TRPV4 had less biteforce attenuation than did normal mice, suggesting that they had less pain. Additionally, in normal mice, TRPV4 expression in trigeminal sensory neurons was greater when inflammation was induced, and this increase in TRPV4 corresponded with greater bite-force attenuation. Furthermore, blockade of TRPV4 expression in normal mice led to reduced bite-force attenuation, similar to that observed in mice lacking TRPV4 (Pain 154, 1295-1304; 2013).

These results suggest that TRPV4 contributes to TMJD-like pain in mice. But lack of TRPV4 does not appear to prevent TMJD-related damage. Bone erosion and jaw inflammation were comparable in normal mice and mice lacking TRPV4. "Remarkably, the damage is the same but not the pain," Liedtke said.

Nevertheless, approaches to block TRPV4 may prove useful in treating the pain associated with TMJD.

Monica Harrington

\section{CHOLESTEROL KEY IN RETI SYNDROME}

A class of cholesterol-lowering drugs called statins decreased Rett syndrome symptoms in a rodent model, suggesting that targeting cholesterol pathways may be key for treating this disorder.

Rett syndrome is a genetic disorder that is X-linked, so it primarily affects girls. The neurological disorder begins to affect development in toddlers, who gradually lose their speech and motor abilities. Some patients also have seizures and severe digestive problems, and most are unable to take care of themselves as adults.

Monica Justice (Baylor College of Medicine, Houston, TX) led a study to search for possible gene targets for the treatment of this disorder. She and her colleagues injected healthy male mice with a chemical called ENU that mutates sperm cells randomly. Then they mated the males to females of the Rett mouse model, which have a mutated Mecp2 (methyl-CpG-binding protein 2) gene. Mecp2 turns other genes on or off by disrupting chromatin.

Next, they screened the offspring of these mice to see which ones had fewer symptoms of Rett syndrome, despite having mothers with the Mecp2 mutation. A group of the offspring performed better on mobility tests and lived longer than most mice with the Mecp2 mutation, indicating that the randomly induced mutations in their genes had 'rescued' them from developing symptoms of Rett syndrome. Justice and her team screened these mice for the gene mutations that modified the effects of Mecp2. A gene called squalene epoxidase (Sqle), which encodes a rate-limiting enzyme in the cholesterol biosynthetic pathway, was the most drug-targetable gene found in the screen (Nat. Genet. published online 28 July 2013; doi:10.1038/ng.2714). The researchers wondered whether inhibition of this pathway was the mechanism that rescued the animals.

Mice with Mecp2 mutations were treated with several different statins. Although statins do not affect Sqle specifically, they interfere with another rate-limiting enzyme in cholesterol metabolism. All of the statins tested improved symptoms in these mice. Similar to the rescued mice in the gene mutation experiment, the statin-treated mice showed improvements in mobility, gross motor function, overall health and lifespan compared to untreated mice with the Mecp2 mutation.

Cholesterol metabolism is also being studied in connection with other neurological diseases, including fragile $X$ syndrome, neurofibromatosis and amyotrophic lateral sclerosis. The next step for the researchers will be to test whether other drugs that affect cholesterol metabolism provide similar results. Furthermore, such drugs must be tested in clinical trials with children before they can be approved for treatment of Rett syndrome. 